\title{
On the Existence of Normal Modes of Damped Discrete-Continuous Systems
}

\author{
H. T. Banks \\ Center for Research in Scientific Computation \\ North Carolina State University \\ Raleigh, NC 27695-8205 \\ Zheng-Hua Luo \\ Dept of Mechanical Engineering \\ Nagaoka University of Technology \\ Nagaoka, Niigata 940-21, Japan \\ L. A. Bergman \\ Dept of Aeronautical and Astronautical Engineering \\ University of Illinois \\ Urbana, IL 61801 \\ D. J. Inman \\ Dept of Engineering Science and Mechanics \\ Virginia Polytechnic Institute \& State University \\ Blacksburg, VA 24061-0219
}

\begin{abstract}
In this paper we investigate a class of combined discrete-continuous mechanical systems consisting of a continuous elastic structure and a finite number of concentrated masses, elastic supports and linear oscillators of arbitrary dimension. After the motion equations for such combined systems are derived, they are formulated as an abstract evolution equation on an appropriately defined Hilbert space. Our main objective is to ascertain conditions under which the combined systems have classical normal modes. Using the sesquilinear form approach, we show that unless some matching conditions are satisfied, the combined systems cannot have normal modes even if Kelvin-Voigt damping is considered.
\end{abstract}




\section{Introduction}

The vibration of distributed parameter structures is a subject of widespread and lasting interest in engineering, and there is a large literature on the subject contributed by both mathematically inclined theorists and practicing structural engineers. As is well-known, the dynamical models for vibrating structures, given by partial differential equations together with appropriate boundary and initial conditions, are essentially infinite dimensional systems. For theoretical convenience and analysis, it is often assumed that the system does not possess damping. The concept of undamped systems not only serves a useful purpose for investigating the intrinsic properties of infinite dimensional systems but can also be loosely justified as an approximation in certain circumstances involving light damping and short time observation of vibrations. But in fact all physical systems do possess damping, no matter how small. Indeed, if a system (even in a vacuum) is set in motion and allowed to vibrate freely, the vibration will eventually die out; the rate of decay depends on the amount of damping. There are several mathematical models representing physical damping. The most often encountered types of damping in vibration studies are linear viscous damping and Kelvin-Voigt damping which are special cases of proportional damping. Viscous damping usually models external friction forces such as air resistance acting on the vibrating structures and is thus called "external damping", while Kelvin-Voigt damping originates from the internal friction of the material of the vibrating structures and is thus called "internal damping" or "material damping".

In system analysis, identification and control, it is convenient and highly desirable that the original partial differential equations together with the boundary conditions be decomposable into an infinite number of uncoupled ordinary differential equations. When possible, this is usually done by using an eigenfunction expansion of the system stiffness operator. Indeed, this is the basic assumption underlying most engineering analyses and testing of complex physical systems, as well as a popular assumption in feedback control design. If a system has this decoupling property, we say that the system has "Normal Modes". For undamped systems, it is widely recognized that a continuous system usually has normal modes. For damped systems, however, the situation is much more complicated. For lumped systems consisting of mass, spring and dampers, it has been shown that the commutability of the mass weighted stiffness operator and damping operator is a necessary and sufficient condition for the existence of normal modes (Caughey, 1960). These results are extended to continuous systems in (Caughey and O'Kelley, 1965) and to continuous systems with special asymmetries in (Inman and Olsen, 1988). In (Meirovitch, 1967), it is shown that a damped continuous mechanical system has normal modes if the damping operator is a linear combination of its 
mass operator and stiffness operator; this is physically a very special case. Mathematically, the condition for the existence of "normal modes" is that one be able to simultaneously diagonalize (through a coordinate transformation) the mass, stiffness and damping operators of the system. This is the standard definition of normal modes as used in both vibration analysis and in vibration testing (Ewins, 1984).

In this paper we investigate a more general a class of mechanical systems, consisting of a continuous structure and a finite number of concentrated masses, elastic supports and oscillators. Such systems are often called "combined dynamical systems" in engineering literature. These include systems arising in the emerging field of smart material structures as well as oft-studied articulated structures (such as those arising in large space structures and robotics applications). Interest in such systems is likewise of long standing, but it has only been in the last several years that methods for the systematic and practical analysis of their vibration, both free and forced, have emerged (see, for example, Bergman and Nicholson, 1985a; Bergman and Nicholson, 1985b; Nicholson and Bergman, 1985; Nicholson and Bergman, 1986a; Nicholson and Bergman, 1986b; Bergman and McFarland, 1988a; Bergman and McFarland, 1988b). While the parallels between the analyses of simple continuous structures and of these combined systems have been recognized for some time (Miles, 1956; Bishop and Johnson, 1960; Lin, 1962; Chen, 1963a; Chen, 1963b; Pan, 1965; Gupta, 1970; Jacquot, 1970; Mead, 1971; Davies and Roger, 1979; Dowell, 1979; Kasprzyk, 1984; Banks, Gates, Rosen, and Wang, 1988), these have not, heretofore, been fully investigated. Since one quickly enters the realm of models with unbounded operators, the analysis is often quite delicate and difficult to carry out with rigor.

We shall be working with the weak form of system dynamic equations by using sesquilinear forms. We believe these are the most natural tools to use for the discussions here because the stiffness and damping operators are essentially differential operators containing delta functions and/or distributional derivatives. Earlier ad hoc approaches (Bergman and McFarland, 1988; McFarland, 1990) to examination of such systems attempted to extend the commutativity of operators conditions (see also Inman and Olsen, 1988) directly to combined systems. Such an approach leads immediately to conceptual difficulties with domains of operators and interpretations of derivatives. To avoid such difficulties we employ a sesquilinear form approach in the context of weak or variational solutions. Using this approach, we shall derive some conditions under which such systems have normal modes. Before we begin work on the general model, we shall look at several special cases. One is a cantilever flexible beam system with piezoceramic patches (the standard elementary model in smart material studies). The other is a cantilever beam with an oscillator attached at some point. The first model arises from control of articulated multidimensional flexible structures using sensor/actuator 
configurations involving piezoceramic patches. In this case, the coefficients of the damping operator as well as the stiffness operator (and even the mass density) contain characteristic functions and thus are not continuous. We shall show that the system generally does not have normal modes even for Kelvin-Voigt damping. For the second system, we shall also show that the system does not possess normal modes except in very special cases where the beam and oscillator are "matched". Based on these observations, we next present a general model for a combined discrete and continuous system and discuss the conditions under which the overall system has normal modes. When Kelvin-Voigt damping is considered, it is found, much like the second case above, that the overall system does not possess normal modes unless some matching conditions are satisfied. These results are interesting because they reveal that a combined discrete-continuous system rarely possesses normal modes even if the continuous system itself does. Thus the usual engineering analyses that begin with the assumption of normal modes are simply not valid and hence not useful in analyzing these very important systems. Despite this, when the system is fully descretized in the usual way via the finite element or assumed modes method, the propensity is, indeed, to invoke the assumption of classical damping, accepting the likelihood of modeling error without a full understanding of its origin or effect.

\section{Motivating Examples}

To motivate the general cases, we first consider three special cases.

Example 1. As the most common example used in the "smart structures" literature, we consider a flat beam subject to transverse vibrations with a pair of piezoceramic patches attached for sensing and actuating. The beam, depicted in Fig.1, is assumed to have length $\ell$, width $b$, thickness $h$ and cantilever end conditions with the fixed end at $x=0$ and free end at $x=\ell$. It is assumed that the beam is homogeneous and constructed from a material which essentially satisfies the Euler-Bernoulli hypothesis for displacement and Kelvin-Voigt damping hypothesis (this means that damping is proportional to strain rate). To control the vibration of the beam, a pair of identical piezoceramic patches are bonded to opposite sides of the beam over the region $x_{1}<x<x_{2}$. The Young's moduli, linear mass densities and damping coefficients for the beam and patch are denoted by $E, E_{p}, \rho, \rho_{p}$ and $C_{d}, C_{d p}$, respectively, with the subscript $p$ denoting patch properties. The patch thickness is denoted by $h_{p}$.

Let $w(t, x)$ denote the transverse displacement of the beam at time $t$ and at position $x$. From fundamental mechanics (force and moment balance for small disturbances and initial 


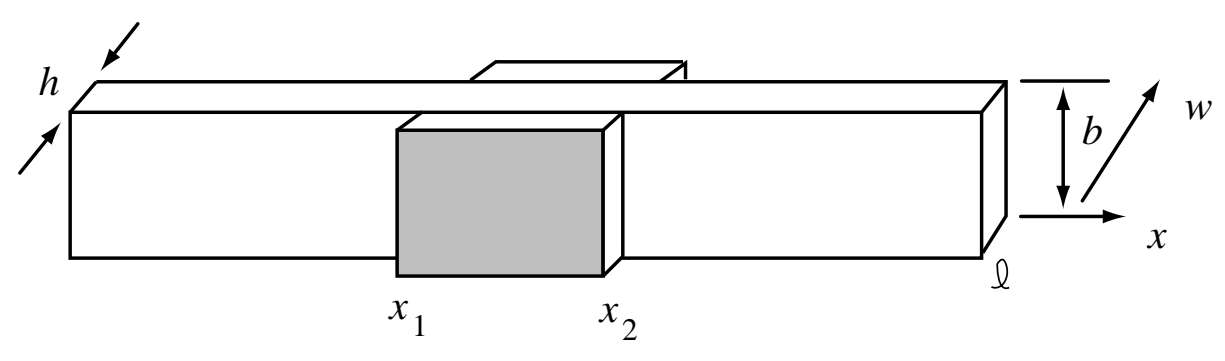

Figure 1: Cantilevered beam with piezoceramic patches

conditions), we know that the dynamic equation governing the free vibrations (no control action) of the beam is given by (Banks, Smith, and Wang, 1996)

$$
\begin{gathered}
\tilde{\rho} \frac{\partial^{2} w}{\partial t^{2}}+\frac{\partial^{3}}{\partial t \partial x^{2}}\left(\widetilde{C_{d} I}(x) \frac{\partial^{2} w}{\partial x^{2}}\right)+\frac{\partial^{2}}{\partial x^{2}}\left(\widetilde{E I}(x) \frac{\partial^{2} w}{\partial x^{2}}\right)=0 \\
w(t, 0)=\frac{\partial w}{\partial x}(t, 0)=\frac{\partial^{2} w(t, \ell)}{\partial x^{2}}=\frac{\partial^{3} w(t, \ell)}{\partial x^{3}}=0
\end{gathered}
$$

where the derivatives are interpreted in the sense of distributions (e.g., see (Banks, Smith, and Wang, 1996) for details as well as experimental validation of the appropriateness of this model for our example) and

$$
\begin{aligned}
\tilde{\rho} & =\rho h b+2 b \rho_{p} h_{p} \chi_{p}(x) \\
\widetilde{C_{d} I}(x) & =C_{d} I+\frac{2 b}{3} C_{d p} a \chi_{p}(x) \\
\widetilde{E I}(x) & =E I+\frac{2 b}{3} E_{p} a \chi_{p}(x)
\end{aligned}
$$

with $I=\frac{h^{3} b}{12}, a=\left(h / 2+h_{p}\right)^{3}-h^{3} / 8$, and $\chi_{p}(x)$ is the characteristic function for $\left[x_{1}, x_{2}\right]$.

It is clear that $\widetilde{E I}(x)$ and $\widetilde{C_{d} I}(x)$ are discontinuous as a function of $x$. Define $V=$ $H_{L}^{2}(0, \ell)=\left\{\phi \in H^{2}(0, \ell) \mid \phi(0)=\phi^{\prime}(0)=0\right\}, H=L^{2}(0, \ell)$ and assume the Gelfand triple construction $V \hookrightarrow H \hookrightarrow V^{*}$ (see Wloka, 1992). Here $H^{2}(0, \ell)$ is the usual complex Sobolov space $W^{2,2}(0, \ell)$. Then the stiffness operator $A: V \rightarrow V^{*}$ and damping operator $B: V \rightarrow V^{*}$ are given by

$$
A=\frac{\partial^{2}}{\partial x^{2}}\left(\widehat{E I}(x) \frac{\partial^{2}}{\partial x^{2}}\right), \quad B=\frac{\partial^{2}}{\partial x^{2}}\left(\widetilde{C_{d} I}(x) \frac{\partial^{2}}{\partial x^{2}}\right)
$$

where the derivatives are interpreted in the distributional sense. Or, more precisely $(A \phi)(\psi)=$ $\left\langle\widetilde{E I} \phi^{\prime \prime}, \psi^{\prime \prime}\right\rangle,(B \phi)(\psi)=\left\langle\widetilde{C_{d} I} \phi^{\prime \prime}, \psi^{\prime \prime}\right\rangle$ for $\phi, \psi \in V,\langle\cdot, \cdot\rangle$ is the complex inner product in $L^{2}(0, \ell)$ and prime denotes spatial differentiation. 
The system (1) can be written thus as (again see (Banks, Smith, and Wang, 1996))

$$
M \ddot{y}(t)+B \dot{y}(t)+A y(t)=0 \text { in } V^{*}
$$

where $M$ is $\tilde{\rho} I d$ with $I d$ the identity on $V$ (or $H$ or $V^{*}$ ). For normal modes one must have a complete orthonormal family $\left\{\phi_{n}\right\}$ satisfying

$$
\begin{aligned}
& \left\langle M \phi_{n}, \phi_{m}\right\rangle=\delta_{n m} \\
& \left(A \phi_{n}\right)\left(\phi_{m}\right)=\lambda_{n} \delta_{n m} \\
& \left(B \phi_{n}\right)\left(\phi_{m}\right)=\tilde{\lambda}_{n} \delta_{n m}
\end{aligned}
$$

where $\delta_{n m}$ is the usual Kronecker delta. In particular, we must have for all $n, m$

$$
\begin{aligned}
\rho h b\left\langle\phi_{n}, \phi_{m}\right\rangle+2 \rho_{p} h_{p} b \int_{x_{1}}^{x_{2}} \phi_{n} \bar{\phi}_{m} & =\delta_{n m} \\
E I\left\langle\phi_{n}^{\prime \prime}, \phi_{m}^{\prime \prime}\right\rangle+\frac{2 b}{3} E_{p} a \int_{x_{1}}^{x_{2}} \phi_{n}^{\prime \prime} \bar{\phi}_{m}^{\prime \prime} & =\lambda_{n} \delta_{n m} \\
C_{d} I\left\langle\phi_{n}^{\prime \prime}, \phi_{m}^{\prime \prime}\right\rangle+\frac{2 b}{3} C_{d p} a \int_{x_{1}}^{x_{2}} \phi_{n}^{\prime \prime} \bar{\phi}_{m}^{\prime \prime} & =\tilde{\lambda}_{n} \delta_{n m} .
\end{aligned}
$$

The first equation just states that $\left\{\phi_{n}\right\}$ is an orthonormal set in $L^{2}(0, \ell)$ with the weighted inner product $\langle\tilde{\rho} \cdot, \cdot\rangle$. Defining $\hat{E}=E I, \hat{C}_{d}=C_{d} I, \hat{E}_{p}=\frac{2 b}{3} E_{p} a, \hat{C}_{d p}=\frac{2 b}{3} C_{d p} a$, we may write the second and third equations as

$$
\mathcal{K}\left(\begin{array}{c}
\left\langle\phi_{n}^{\prime \prime}, \phi_{m}^{\prime \prime}\right\rangle \\
\int_{x_{1}}^{x_{2}} \phi_{n}^{\prime \prime} \bar{\phi}_{m}^{\prime \prime}
\end{array}\right)=\left(\begin{array}{c}
\lambda_{n} \delta_{n m} \\
\hat{\lambda}_{n} \delta_{n m}
\end{array}\right)
$$

where $\mathcal{K}=\left[\begin{array}{cc}\hat{E} & \hat{E}_{p} \\ \hat{C}_{d} & \hat{C}_{d p}\end{array}\right]$. Thus, if $\mathcal{K}$ us invertible, that is, if we do not have existence of a $k \neq 0$ such that $\left(\hat{E}, \hat{E}_{p}\right)=k\left(\hat{C}_{d}, \hat{C}_{d p}\right)$, we find that

$$
\left(\begin{array}{c}
\left\langle\phi_{n}^{\prime \prime}, \phi_{m}^{\prime \prime}\right\rangle \\
\int_{x_{1}}^{x_{2}} \phi_{n}^{\prime \prime} \bar{\phi}_{m}^{\prime \prime}
\end{array}\right)=\mathcal{K}^{-1}\left(\begin{array}{c}
\lambda_{n} \delta_{n m} \\
\tilde{\lambda}_{n} \delta_{n m}
\end{array}\right)=\left(\begin{array}{c}
\gamma_{n} \delta_{n m} \\
\tilde{\gamma}_{n} \delta_{n m}
\end{array}\right)
$$

for all $n, m$. In particular, this implies that $\left\langle\tilde{\rho} \phi_{n}, \phi_{m}\right\rangle=\delta_{n m},\left\langle\phi_{n}^{\prime \prime}, \phi_{m}^{\prime \prime}\right\rangle=\gamma_{n} \delta_{n m}$ and the $\left\{\phi_{n}\right\}$ satisfy the boundary conditions for a cantilevered beam. That is, the $\left\{\phi_{n}\right\}$ form a complete orthonormal family for an undamped beam with mass density $\tilde{\rho}$ and stiffness $E I=1$ (such a family is completely determined by the beam parameters and boundary conditions in the usual manner). However, the second equation arising from (4) yields that this set $\left\{\phi_{n}\right\}$ must also satisfy $\int_{x_{1}}^{x_{2}} \phi_{n}^{\prime \prime} \bar{\phi}_{m}^{\prime \prime}=0$ for $n \neq m$, a condition that does not in general hold for an arbitrary patch on $\left[x_{1}, x_{2}\right] \subset(0, \ell)$. Hence the assumption of invertibility of $\mathcal{K}$ leads to a contradiction. 
Thus if we are to have a set of normal modes for the cantilevered beam with patches, we must have that $\left(\hat{E}, \hat{E}_{p}\right)=k\left(\hat{C}_{d}, \hat{C}_{d p}\right)$ for some $k \neq 0$. This condition (while an extremely rare and physically unreasonable condition in general) is clearly also sufficient for normal modes. Thus we have argued

Theorem 1. A necessary and sufficient condition that the system (1) have normal modes is that there exist $k>0$ such that $\left(\hat{E}, \hat{E}_{p}\right)=k\left(\hat{C}_{d}, \hat{C}_{d p}\right)$ where $\hat{E} \equiv E I, \hat{E}_{p}=\frac{2 b}{3} E_{p} a, \hat{C}_{d}=C_{d} I$, $\hat{C}_{d p}=\frac{2 b}{3} C_{d p} a$.

It is interesting to observe that while the standard "smart structure", i.e., system (1) with piezoceramic patches does not in general possess normal modes, the system without the patches and with homogeneous material does have normal modes for Kelvin-Voigt damping is present.

Example 2. Consider a cantilever beam with an oscillator attached to the beam at point $\alpha$, as illustrated in Fig.2. Again, consider Kelvin-Voigt damping. Let the notations for the beam be the same as in Example 1, except that now $\widetilde{E I}=E I$ and $\widehat{C_{d} I}=C_{d} I$ are constants.

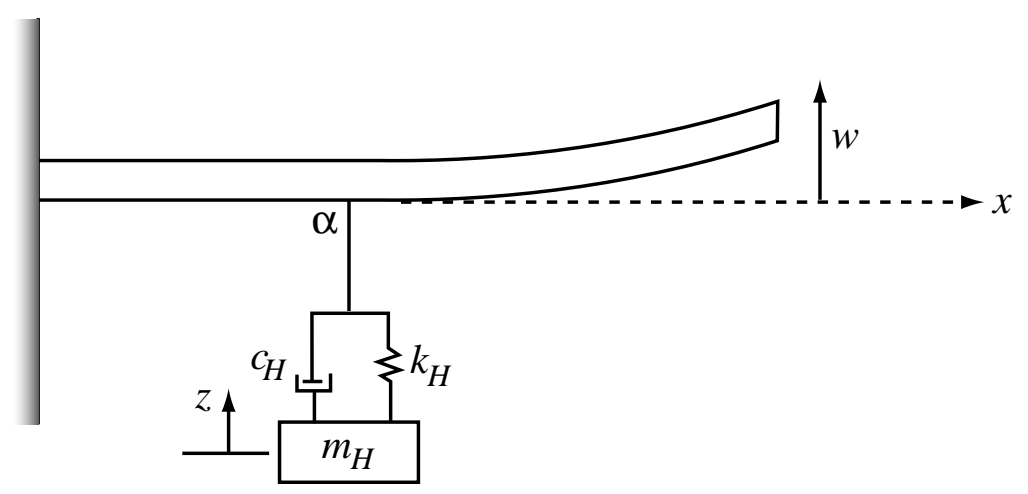

Figure 2: Cantilevered beam with oscillator attached

Let the mass, damping and spring coefficients of the oscillator be denoted by $m_{H}, c_{H}$ and $k_{H}$, respectively. Let $w(t, x)$ denote the displacement of the beam and let $z(t)$ denote the 
displacement of the mass of the oscillator. It is assumed that both are measured from the equilibrium state. Then the dynamical system of the composite structure is given by

$$
\begin{aligned}
& \rho \ddot{w}(t, x)+C_{d} I \dot{w}^{\prime \prime \prime \prime}(t, x)+E I w^{\prime \prime \prime \prime}(t, x) \\
& \quad=\left[c_{H}(\dot{z}(t)-\dot{w}(t, x))+k_{H}(z(t)-w(t, x))\right] \delta(x-\alpha), \quad 0<x<\ell, \\
& m_{H} \ddot{z}(t)+c_{H}(\dot{z}(t)-\dot{w}(t, \alpha))+k_{H}(z(t)-w(t, \alpha))=0, \\
& w(0, x)=w_{0}(x), \dot{w}(0, x)=w_{1}(x), z(0)=z_{0}, \dot{z}(0)=z_{1},
\end{aligned}
$$

where $\delta(x-\alpha)$ is the Dirac Delta distribution, and hence $\phi(\alpha)=\int_{0}^{\ell} \phi(x) \delta(x-\alpha) d x$ for $\phi \in H^{1}(0, \ell)$, and $w_{0}, w_{1}, z_{0}, z_{1}$ are given initial data.

To proceed further, we define a complex Hilbert space $H=L_{2}(0, \ell) \times \mathbb{C}$ with inner product

$$
<y_{1}, y_{2}>=\int_{0}^{\ell} w_{1}(x) \bar{w}_{2}(x) d x+z_{1} \bar{z}_{2}, \quad \text { for } y_{1}=\left(w_{1}, z_{1}\right), y_{2}=\left(w_{2}, z_{2}\right) \in H
$$

Define a Hilbert space $V=\left\{(w, z) \in H^{2}(0, \ell) \times \mathbb{C} \mid w(0)=w^{\prime}(0)=0\right\}$ with inner product induced norm

$$
\|(w, z)\|_{V}^{2}=\int_{0}^{\ell} w^{\prime \prime}(x) \bar{w}^{\prime \prime}(x) d x+(z-w(\alpha))(\bar{z}-\bar{w}(\alpha)) .
$$

Let $V \hookrightarrow H \hookrightarrow V^{*}$ again be the usual Gelfand triple formulation.

Define a stiffness operator $A: V \rightarrow V^{*}$ by

$$
A\left(\begin{array}{c}
w \\
z
\end{array}\right)=\left(\begin{array}{c}
E I w^{\prime \prime \prime \prime}(x)+k_{H}(w(x)-z) \delta(x-\alpha) \\
k_{H}(z-w(\alpha))
\end{array}\right)
$$

and a damping operator $B: V \rightarrow V^{*}$ by

$$
B\left(\begin{array}{c}
w \\
z
\end{array}\right)=\left(\begin{array}{c}
C_{d} I w^{\prime \prime \prime \prime}(x)+c_{H}(w(x)-z) \delta(x-\alpha) \\
c_{H}(z-w(\alpha))
\end{array}\right),
$$

where again the derivatives and Delta operator are interpreted in the distributional sense. Then Equation (5) can be written as the abstract second-order initial value problem

$$
\begin{aligned}
& M \ddot{y}(t)+B \dot{y}(t)+A y(t)=0 \quad \text { in } V^{*}, t>0, \\
& y(0)=y_{0}, \quad \dot{y}(0)=y_{1}
\end{aligned}
$$

where $y=(w, z)^{T}$ and $M y=\left(\rho w, m_{H} z\right)^{T}, y_{0}=\left(w_{0}, z_{0}\right), y_{1}=\left(w_{1}, z_{1}\right)$.

Let $\sigma_{A}\left(y_{1}, y_{2}\right): V \times V \rightarrow \mathbb{C}$ be the symmetric sesquilinear form given by

$$
\sigma_{A}\left(y_{1}, y_{2}\right)=E I \int_{0}^{\ell} w_{1}^{\prime \prime}(x) \bar{w}_{2}^{\prime \prime}(x) d x+k_{H}\left(w_{1}(\alpha)-z_{1}\right)\left(\bar{w}_{2}(\alpha)-\bar{z}_{2}\right)
$$


for $y_{1}=\left(w_{1}, z_{1}\right), y_{2}=\left(w_{2}, z_{2}\right) \in V$.

Similarly, let $\sigma_{B}$ be the sesquilinear form which has exactly the same form as $\sigma_{A}$ except the constants $E I$ and $k_{H}$ are replaced respectively by $C_{d} I$ and $c_{H}$. At this point, it is easy to show that

$$
\sigma_{A}\left(y_{1}, y_{2}\right)=<A y_{1}, y_{2}>_{V^{*}, V} \text { and } \sigma_{B}\left(y_{1}, y_{2}\right)=<B y_{1}, y_{2}>_{V^{*}, V}
$$

where $\langle\cdot, \cdot\rangle_{V^{*}, V}$ denotes the usual duality pairing (Banks, Smith, and Wang, 1996; Wloka, 1992). Furthermore, we have the following lemma.

Lemma 1. For any $y_{1}, y_{2} \in V$, there exist positive constants $\gamma$ and $c$ such that

i) $\left|\sigma_{A}\left(y_{1}, y_{2}\right)\right| \leq c\left\|y_{1}\right\|_{V}\left\|y_{2}\right\|_{V}$, i.e., $\sigma_{A}$ is $V$-continuous.

ii) $\sigma_{A}\left(y_{1}, y_{1}\right) \geq \gamma\left\|y_{1}\right\|_{V}^{2}$, i.e., $\sigma_{A}$ is $V$-elliptic.

The proof of this lemma is obvious and is thus omitted.

Similarly, we can show that $\sigma_{B}$ has the same properties as stated in Lemma 1.

Using these notations, we are able to write the equation of (5) as

$$
<M \ddot{y}(t), \Phi>+\sigma_{A}(\dot{y}(t), \Phi)+\sigma_{B}(y(t), \Phi)=0, \quad \text { for all } \Phi \in V \text {. }
$$

This is the precise meaning of the equation in (6) which is called the weak form or variational form of system (5). We can rewrite equation (8) as an equivalent first order vector system, or leave it in second order form. In either case, there are basic abstract results (see Chap. 4 of (Banks, Smith, and Wang, 1996)) for such systems that guarantee the existence of a unique solution in an appropriate sense. We are more interested here in the conditions under which there exist normal modes.

Theorem 2. There exist normal modes for system (6) if and only if there exists a constant $k>0$ such that

$$
\left(\begin{array}{c}
E I \\
k_{H}
\end{array}\right)=k\left(\begin{array}{c}
C_{d} I \\
c_{H}
\end{array}\right)
$$

Proof: Condition (9) is obviously sufficient. We need only to show the necessity. Let $\Phi_{n}$ be the generalized orthonormal eigenvectors of the operator $A$ such that

$$
<M \Phi_{n}, \Phi_{m}>=\delta_{n m}, \text { for all } n, m
$$

That is, the $\left\{\Phi_{n}\right\}$ simultaneously diagonalize $M$ and $A$. It is shown in the Appendix how to uniquely solve this eigenvalue problem. If there exist normal modes for $(6)$, then it is necessary that these $\left\{\Phi_{n}\right\}$ also diagonalize $B$, that is,

$$
\begin{aligned}
& \sigma_{A}\left(\Phi_{n}, \Phi_{m}\right)=0, \\
& \sigma_{B}\left(\Phi_{n}, \Phi_{m}\right)=0, \quad \text { for all } \Phi_{n}=\left(w_{n}, z_{n}\right), \Phi_{m}=\left(w_{m}, z_{m}\right), \text { for all } n \neq m
\end{aligned}
$$


This means that

$$
\begin{aligned}
& E I \int_{0}^{\ell} w_{n}^{\prime \prime}(x) \bar{w}_{m}^{\prime \prime}(x) d x+k_{H}\left(z_{n}-w_{n}(\alpha)\right)\left(\bar{z}_{m}-\bar{w}_{m}(\alpha)\right)=0 \\
& C_{d} I \int_{0}^{\ell} w_{n}^{\prime \prime}(x) \bar{w}_{m}^{\prime \prime}(x) d x+c_{H}\left(z_{n}-w_{n}(\alpha)\right)\left(\bar{z}_{m}-\bar{w}_{m}(\alpha)\right)=0,
\end{aligned}
$$

for all $n \neq m$. Suppose condition (9) does not hold. Then from these two equations, we have

$$
\begin{gathered}
\int_{0}^{\ell} w_{n}^{\prime \prime}(x) \bar{w}_{m}^{\prime \prime}(x) d x=0 \\
\left(z_{n}-w_{n}(\alpha)\right)\left(\bar{z}_{m}-\bar{w}_{m}(\alpha)\right)=0,
\end{gathered}
$$

for all $n \neq m$, which implies $z_{n}=w_{n}(\alpha)$ or $z_{m}=w_{m}(\alpha)$, for all $n \neq m$. Therefore, there exists at most one integer $n_{1}$ such that $z_{n_{1}} \neq w_{n_{1}}(\alpha)$ and $z_{n}=w_{n}(\alpha)$ for all $n$ except $n=n_{1}$. Since $\Phi_{n}(x)=\left(\begin{array}{c}w_{n}(x) \\ z_{n}\end{array}\right)$ are the eigenfunctions of $A$ which also diagonalize $M$, the equation

$$
A \Phi_{n}(x)=\lambda_{n} M \Phi_{n}(x)
$$

must be satisfied for some scalar $\lambda_{n}$. This is equivalent to

$$
\begin{gathered}
E I w_{n}^{\prime \prime \prime \prime}(x)+k_{H}\left(w_{n}(\alpha)-z_{n}\right) \delta(x-\alpha)=\rho \lambda_{n} w_{n}(x) \\
k_{H}\left(z_{n}-w_{n}(\alpha)\right)=m_{H} \lambda_{n} z_{n} \\
w_{n}(0)=w_{n}^{\prime}(0)=w_{n}^{\prime \prime}(\ell)=w_{n}^{\prime \prime \prime}(\ell)=0 .
\end{gathered}
$$

Since $z_{n}=w_{n}(\alpha)$ for $n \neq n_{1}$, the second equation above gives $z_{n}=0$ for all $n \neq n_{1}$ because $\lambda_{n} \neq 0$. Hence $w_{n}(\alpha)=0$ for all $n \neq n_{1}$. Thus, equation (10) reduces to

$$
\begin{gathered}
E I w_{n}^{\prime \prime \prime \prime}(x)=\rho \lambda_{n} w_{n}(x) \\
w_{n}(x)=w_{n}^{\prime}(0)=w_{n}^{\prime \prime}(\ell)=w_{n}^{\prime \prime \prime}(\ell)=0, \quad n \neq n_{1},
\end{gathered}
$$

which is exactly the eigenvalue problem for a cantilever beam with density $\rho$, stiffness $E I$ without any attached oscillators. Such a set $\left\{w_{n}\right\}$ is determined uniquely (independent of $\alpha$ ) and does not satisfy $w_{n}(\alpha)=0$ for all $n \neq n_{1}$.

Thus condition (9) is necessary for the beam with oscillator system to have normal modes. When condition (9) is satisfied, we say that the beam and the oscillator are "matched". Since they are rarely matched for arbitrarily given oscillators, the overall system rarely has normal modes.

Remark: Consider the special case where the oscillator is attached at the tip end, i.e., $\alpha=\ell$. In this case, the dynamic equations can be written as follows (using force balance notions on the states $y(t)=(w(t, x), w(t, \ell), z(t)))$

$$
\rho \ddot{w}(t, x)+C_{d} I \dot{w}^{\prime \prime \prime \prime}(t, x)+E I w^{\prime \prime \prime \prime}(t, x)=0, \quad 0<x<\ell,
$$




$$
\begin{gathered}
-C_{d} I \dot{w}^{\prime \prime \prime}(t, \ell)-E I w^{\prime \prime \prime}(t, \ell)=c_{H}(\dot{z}(t)-\dot{w}(t, \ell))+k_{H}(z(t)-w(t, \ell)) \\
m_{H} \ddot{z}(t)+c_{H}(\dot{z}(t)-\dot{w}(t, \ell))+k_{H}(z(t)-w(t, \ell))=0 .
\end{gathered}
$$

The second equation above can be formally obtained by integrating the first equation of (5) from $\ell^{-}$to $\ell^{+}$, noting that $w^{\prime \prime \prime}\left(t, \ell^{+}\right)=0, \int_{\ell^{-}}^{\ell^{+}} \ddot{w}(t, x) d x=0$, and then setting $\ell^{-}=\ell$. This model is extensively studied in existing literature (see, for example, (Banks, Gates, Rosen, and Wang, 1988)). It should be noted that there exists an essential difference between equation (5) and equation (11). In equation (11), w( $t, \ell)$ is an independent variable. That is to say, $(w(t, x), w(t, \ell), z(t))$ is chosen as state variable if we wish to write equation (11) in the abstract form as before. This is in sharp contrast with equation (5) where $w(t, \alpha)$ is not an independent variable. In that case only $y(t)=(w(t, x), z(t))$ was chosen as the state variable. Since the mathematical formulation is thus somewhat different for discrete elements attached to the free end of a beam, we shall assume that the discrete elements such as oscillators are attached only at the interior points of continuous structures in the next section.

In (Inman, 1989, p281) it is shown formally (not rigorously) that the modal analysis of the system of Fig. 2 "effectively couples the design problem so that passive control cannot be performed on a per mode basis." It is also noted in (Inman, 1989) from an analysis of (Bergman and Nicholson, 1985b) that proportionality results only if $\lambda_{n} \lambda_{m}=$ constant for all $m, n$. This, of course, is not possible with the exception of perhaps one mode. In (Bellos, 1989 ) it is shown experimentally that in fact only one mode of the system of Fig. 2 exhibits a normal mode behavior.

Example 3. Let us now consider the situation where an elastic support is attached to the beam at $x=\beta$. Let $k_{e}$ and $c_{e}$ denote respectively the spring and damping coefficients of the elastic support. Using the notations defined in Example 2, we are able to write the dynamical equations governing the free vibrations of the beam as follows:

$$
\begin{gathered}
\rho \ddot{w}(t, x)+C_{d} I \dot{w}^{\prime \prime \prime \prime}(t, x)+E I w^{\prime \prime \prime \prime}(t, x) \\
+k_{e} w(t, x) \delta(x-\beta)+c_{e} \dot{w}(t, x) \delta(x-\beta)=0, \quad 0<x<\ell .
\end{gathered}
$$

Let $V=\left\{w \in H^{2}(0, \ell) \mid w(0)=w^{\prime}(0)=0\right\}$. Define $A: V \rightarrow V^{*}$ and $B: V \rightarrow V^{*}$ by

$$
A w(x)=E I w^{\prime \prime \prime \prime}(x)+k_{e} w(x) \delta(x-\beta), \quad B w(x)=C_{d} I w^{\prime \prime \prime \prime}(x)+c_{e} w(x) \delta(x-\beta) .
$$

Let $\sigma_{A}$ and $\sigma_{B}$ be the sesquilinear forms associated with $A$ and $B$. We show that for the system to have normal modes, it is necessary and sufficient that there exists a constant $k$ such that

$$
\left(\begin{array}{c}
E I \\
k_{e}
\end{array}\right)=k\left(\begin{array}{c}
C_{d} I \\
c_{e}
\end{array}\right)
$$


The proof of this claim is similar to but simplier than that in Example 2. So we only provide an outline. Let $\left\{w_{n}\right\}$ be the generalized orthogonal eigenfunctions of $A$. To have normal modes, it is necessary that

$$
\begin{aligned}
& \sigma_{A}\left(w_{n}, w_{m}\right)=0, \\
& \sigma_{B}\left(w_{n}, w_{m}\right)=0, \quad \text { for all } n \neq m,
\end{aligned}
$$

which means

$$
\begin{aligned}
E I \int_{0}^{\ell} w_{n}^{\prime \prime}(x) \bar{w}_{m}^{\prime \prime}(x) d x+k_{e} w_{n}(\beta) \bar{w}_{m}(\beta) & =0, \\
C_{d} I \int_{0}^{\ell} w_{n}^{\prime \prime}(x) \bar{w}_{m}^{\prime \prime}(x) d x+c_{e} w_{n}(\beta) \bar{w}_{m}(\beta) & =0, \quad \text { for all } n \neq m .
\end{aligned}
$$

Therefore, if $\left(E I, k_{e}\right) \neq k\left(C_{d} I, c_{e}\right)$, then

$$
\begin{gathered}
\int_{0}^{\ell} w_{n}^{\prime \prime}(x) \bar{w}_{m}^{\prime \prime}(x) d x=0, \\
w_{n}(\beta) \bar{w}_{m}(\beta)=0,
\end{gathered}
$$

which implies $w_{n}(\beta)=0$ for all $n$, except possibly one integer $n_{1}$. But then, the eigenvalue problem $A w_{n}(x)=\lambda_{n} w_{n}(x)$ is reduced to

$$
\begin{gathered}
E I w_{n}^{\prime \prime \prime \prime}(x)=\lambda_{n} w_{n}(x), \\
w_{n}(0)=w_{n}^{\prime}(0)=w_{n}^{\prime \prime}(\ell)=w_{n}^{\prime \prime \prime}(\ell)=0, \quad n \neq n_{1}
\end{gathered}
$$

which is the eigenvalue problem for a free beam without any attachments. For the latter, the eigenfunctions are already determined and they cannot satisfy the constraint $w_{n}(\beta)=0$ for

all $n \neq n_{1}$ and arbitrary $\beta$. This contradiction shows that $\left(E I, k_{e}\right)=k\left(C_{d} I, c_{e}\right)$ is necessary for the system to have normal modes.

\section{Normal Modes of Combined Discrete-Continuous Sys- tems}

In this section we generalize the findings of the specific examples of the previous section to treat combined systems that include a multi-dimensional distributed parameter structural member, such as a beam, plate or shell, subject to transverse vibrations, to which a finite number of discrete systems have been attached. Other vibrating structures, such as shafts in torsion, are readily treated by the ideas to be presented here.

We consider a typical two-dimensional planar member, occupying a region denoted by $\Omega$ with a boundary of $\partial \Omega$ arbitrary shape. This structure, which has area mass density $\rho$, is 
assumed to undergo transverse displacements $w\left(t, x_{1}, x_{2}\right)$ that can be described by the usual linear theory of infinitesimal elasticity. We further assume that

Assumption (H1): Some part of the boundary is clamped and the natural boundary conditions on the remaining part are such that the stiffness operator is self-adjoint in some appropriate Hilbert spaces to be defined below.

This is a reasonable assumption for a significant number of realistical physical systems. To treat the problem in a unified form, we further assume in the following that there are no attachments of the discrete subsystems on the boundary. However,as noted in the remark in the last section, the case where attachments do exist on the boundary can also be treated.

A total of $\mathcal{N}$ discrete subsystems is attached to the continuous member at the points $\xi_{i}, i=1, \cdots, \mathcal{N}$ in $\Omega$. Specifically, let $\mathcal{N}_{e}$ be the number of elastic supports (linear springs), $\mathcal{N}_{m}$ the number of concentrated masses, and $\mathcal{N}_{0}$ the number of lumped parameter linear oscillators in the combined system, so that $\mathcal{N}=\mathcal{N}_{e}+\mathcal{N}_{m}+\mathcal{N}_{o}$. Examples of such discrete systems are depicted in Fig.3. The $i$ th elastic support has stiffness $k_{i}$ and includes a dashpot with viscous damping coefficient $c_{i}$; the $i$ th concentrated mass is of mass $m_{i}$; and the $i$ th discrete oscillator has $N=N(i)$ degrees of freedom, with stiffness $k_{i j}$, damping coefficients $c_{i j}$, and masses $m_{i j}, j=1, \cdots, N(i)$. The displacement of the $j$ th mass of the $i$ th oscillator is denoted by $z_{i j}(t)$, and the displacements of all the masses of this oscillator can be written as the vector

$$
z_{i}(t)=\left(z_{i 1}(t), \cdots, z_{i N(i)}(t)\right)^{T}, i=1, \cdots, \mathcal{N}_{o}
$$

Since in our discussions we are only concerned with the existence of normal modes of the basic system, we do not assume that any specific external forces are applied to the system.

Let $w(t, x)$ denote the displacement of the continuous structure at time $t$ and position $x$. Let $L_{K}$ and $L_{C}$ denote respectively the stiffness and damping operators of the continuous structure. We assume the following:

Assumption (H2): $L_{C}$ is proportional to $L_{K}$.

We assume this because we already know the continuous system alone has normal modes under this condition and our objective here is to see what happens for the combined discretecontinuous systems under this same condition. It is clear that if we consider Kelvin-Voigt damping in a homogeneous continuous structure, then the above assumption is satisfied automatically. Kelvin-Voigt damping is a proportional strong damping that is most likely to allow the system to have normal modes. In other words, if a system does not have normal modes when Kelvin-Voigt damping is considered, then it is most unlikely to have normal modes for other kinds of damping. 


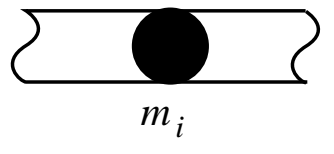

(a)

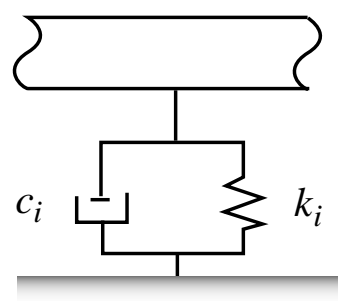

(b)

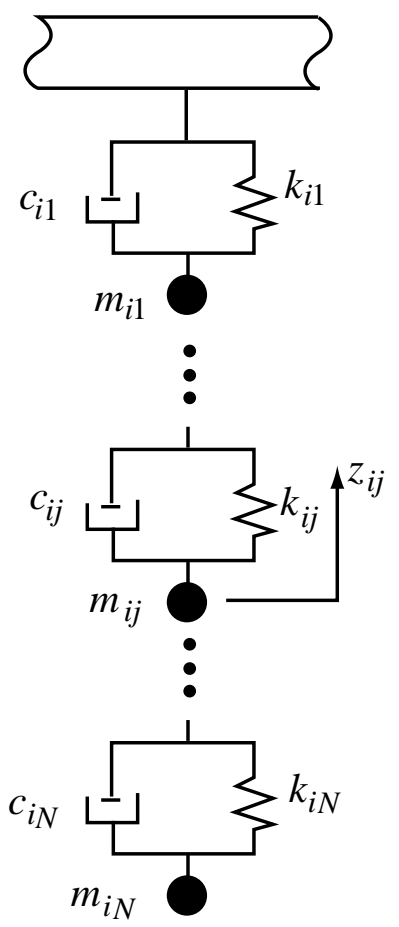

(c)

Figure 3: Examples of attached discrete systems

Using these notations, the free vibration of the continuous member is governed by the equation

$$
\rho \ddot{w}(t, x)+L_{C} \dot{w}(t, x)+L_{K} w(t, x)=\sum_{i=1}^{\mathcal{N}} F_{i}(t) \delta\left(x-\xi_{i}\right)
$$

where $F_{i}(t)$ represent reaction forces exerted on the continuous structure by the discrete subsystems. For an elastic support, they are given by

$$
F_{i}(t)=-k_{i} w\left(t, \xi_{i}\right)-c_{i} \dot{w}\left(t, \xi_{i}\right)
$$

for a concentrated mass,

$$
F_{i}(t)=-m_{i} \ddot{w}\left(t, \xi_{i}\right)
$$

and for an oscillator.

$$
F_{i}(t)=k_{i 1}\left[z_{i 1}(t)-w\left(t, \xi_{i}\right)\right]+c_{i 1}\left[\dot{z}_{i 1}(t)-\dot{w}\left(t, \xi_{i}\right)\right]
$$




$$
=-\sum_{j=1}^{N(i)} m_{i j} \ddot{z}_{i j}(t)
$$

The dynamical equations of the $i$ th oscillator subsystems are given by

$$
\begin{gathered}
m_{i 1} \ddot{z}_{i 1}(t)+c_{i 1}\left[\dot{z}_{i 1}(t)-\dot{w}\left(t, \xi_{i}\right)\right]+c_{i 2}\left[\dot{z}_{i 1}(t)-\dot{z}_{i 2}(t)\right] \\
+k_{i 1}\left[z_{i 1}(t)-w\left(t, \xi_{i}\right)\right]+k_{i 2}\left[z_{i 1}(t)-z_{i 2}(t)\right]=0 \\
m_{i j} \ddot{z}_{i j}(t)+c_{i j}\left[\dot{z}_{i j}(t)-\dot{z}_{i(j-1)}(t)\right]+c_{i(j+1)}\left[\dot{z}_{i j}(t)-\dot{z}_{i(j+1)}(t)\right] \\
+k_{i j}\left[z_{i j}(t)-z_{i(j-1)}\right]+k_{i(j+1)}\left[z_{i j}(t)-z_{i(j+1)}(t)\right]=0, \\
\vdots \\
m_{i N(i)} \ddot{z}_{i N(i)}(t)+c_{i N(i)}\left[\dot{z}_{i N(i)}(t)-\dot{z}_{i(N(i)-1)}(t)\right]+k_{i N(i)}\left[z_{i N(i)}(t)-z_{i(N(i)-1)}(t)\right]=0 .
\end{gathered}
$$

Motivated by Example 2, we wish to write the coupled equations (12) and (16) in a compact form similar to (6). For this purpose, let us define a Hilbert space

$$
H=L^{2}(\Omega) \times \mathbb{C}^{N(1)} \times \cdots \times \mathbb{C}^{N\left(\mathcal{N}_{0}\right)}
$$

with inner products

$$
<U, Y>=\int_{\Omega} u(x) \bar{y}(x) d x+u_{1} \bar{y}_{1}+\cdots+u_{\mathcal{N}_{o}} \bar{y}_{\mathcal{N}_{o}}
$$

for $U(x)=\left(u(x), u_{1}, \cdots, u_{\mathcal{N}_{o}}\right), Y(x)=\left(y(x), y_{1}, \cdots, y_{\mathcal{N}_{o}}\right) \in H$. We also define operators $\hat{\rho}$ and $\hat{L}_{K}$ by

$$
\hat{\rho} U(x)=\left(\begin{array}{c}
{\left[\rho+\sum_{i=1}^{\mathcal{N}_{m}} m_{i} \delta\left(x-\xi_{i}\right)\right] u(x)} \\
M_{1} u_{1} \\
\vdots \\
M_{\mathcal{N}_{o}} u_{\mathcal{N}_{o}}
\end{array}\right)
$$

and

$$
\hat{L}_{K} U(x)=\left(\begin{array}{c}
L_{K} u(x)+\sum_{i=1}^{\mathcal{N}_{o}} k_{i 1}\left(u\left(\xi_{i}\right)-u_{i 1}\right) \delta\left(x-\xi_{i}\right)+\sum_{i=1}^{\mathcal{N}_{e}} k_{i} u\left(\xi_{i}\right) \delta\left(x-\xi_{i}\right) \\
K_{1} u_{1} \\
\vdots \\
K_{\mathcal{N}_{o}} u_{\mathcal{N}_{o}}
\end{array}\right),
$$

where

$$
M_{i}=\operatorname{diag}\left(m_{i j}\right), j=1, \ldots, N(i), i=1, \cdots, \mathcal{N}_{o}
$$

and

$$
K_{i} u_{i}=\left(\begin{array}{c}
k_{i 1}\left(u_{i 1}-u\left(\xi_{i}\right)\right)+k_{i 2}\left(u_{i 1}-u_{i 2}\right) \\
\vdots \\
k_{i N(i)}\left(u_{i N(i)}-u_{i(N(i)-1)}\right)
\end{array}\right), i=1, \cdots, \mathcal{N}_{\circ}
$$


The operator $\hat{L}_{C}$ can also be defined just in the same way as $\hat{L}_{K}$ except that $L_{K}$ and $k_{i j}$ in $\hat{L}_{K}$ should be replaced by $L_{C}$ and $c_{i j}$ respectively.

With these definitions, we can formally write equations (12)-(16) in an abstract form on the Hilbert space $H$ by

$$
\hat{\rho} \ddot{W}(t)+\hat{L}_{C} \dot{W}(t)+\hat{L}_{K} W(t)=0,
$$

for $W(t)=\left(w(t, \cdot), z_{1}(t), \cdots, z_{\mathcal{N}_{0}}(t)\right)$.

Next, let us define a Hilbert space

$$
V=H_{L}^{2}(\Omega) \times \mathbb{C}^{N(1)} \times \cdots \times \mathbb{C}^{N\left(\mathcal{N}_{o}\right)}
$$

where $H_{L}^{2}(\Omega)$ is a space of all functions which are twice differentiable in $L^{2}(\Omega)$ and satisfy the clamped boundary conditions on $\partial \Omega$. The inner product in $V$ can be defined as a summation of the inner product in $H_{L}^{2}(\Omega)$ and the inner products in $\mathbb{C}^{N(i)}, i=1, \cdots, \mathcal{N}_{o}$.

For $U(x)=\left(u(x), u_{1}, \cdots, u_{\mathcal{N}_{o}}\right), Y(x)=\left(y(x), y_{1}, \cdots, y_{\mathcal{N}_{o}}\right)$, we define a sesquilinear form by

$$
\begin{aligned}
& \hat{\sigma}_{K}(U, Y)=\left\langle\hat{L}_{K} U, Y\right\rangle \\
& =\sigma_{K}(u, y)+\sum_{i=1}^{\mathcal{N}_{e}} k_{i} u\left(\xi_{i}\right) \bar{y}\left(\xi_{i}\right) \\
& +\sum_{i=1}^{\mathcal{N}_{o}} k_{i 1}\left(u\left(\xi_{i}\right)-u_{i 1}\right) \bar{y}\left(\xi_{i}\right) \\
& +\left(K_{1} u_{1}\right) \bar{y}_{1}+\cdots+\left(K_{\mathcal{N}_{0}} u_{\mathcal{N}_{o}}\right) \bar{y}_{\mathcal{N}_{o}} \\
& =\sigma_{K}(u, y)+\sum_{i=1}^{\mathcal{N}_{e}} k_{i} u\left(\xi_{i}\right) \bar{y}\left(\xi_{i}\right) \\
& +\sum_{i=1}^{\mathcal{N}_{0}} k_{i 1}\left(u_{i 1}-u\left(\xi_{i}\right)\right)\left(\bar{y}_{i 1}-\bar{y}\left(\xi_{i}\right)\right) \\
& +\sum_{i=1}^{\mathcal{N}_{o}} k_{i 2}\left(u_{i 2}-u_{i 1}\right)\left(\bar{y}_{i 2}-\bar{y}_{i 1}\right) \\
& \vdots \\
& +\sum_{i=1}^{\mathcal{N}_{0}} k_{i N(i)}\left(u_{i N(i)}-u_{i(N(i)-1)}\right)\left(\bar{y}_{i N(i)}-\bar{y}_{i(N(i)-1)}\right)
\end{aligned}
$$

where $\sigma_{K}(\cdot, \cdot)$ is the sesquilinear form associated with $L_{K}$.

Similarly, we can define an sesquilinear form associated with the damping operator $\hat{L}_{C}$ as follows:

$$
\begin{aligned}
\hat{\sigma}_{C}(U, Y) & =\left\langle\hat{L}_{C} U, Y>\right. \\
& =\sigma_{C}(u, y)+\sum_{i=1}^{\mathcal{N}_{e}} c_{i} u\left(\xi_{i}\right) \bar{y}\left(\xi_{i}\right)
\end{aligned}
$$




$$
\begin{aligned}
& +\sum_{i=1}^{\mathcal{N}_{o}} c_{i 1}\left(u_{i 1}-u\left(\xi_{i}\right)\right)\left(\bar{y}_{i 1}-\bar{y}\left(\xi_{i}\right)\right) \\
& +\sum_{i=1}^{\mathcal{N}_{o}} c_{i 2}\left(u_{i 2}-u_{i 1}\right)\left(\bar{y}_{i 2}-\bar{y}_{i 1}\right) \\
& \vdots \\
& +\sum_{i=1}^{\mathcal{N}_{o}} c_{i N(i)}\left(u_{i N(i)}-u_{i(N(i)-1)}\right)\left(\bar{y}_{i N(i)}-\bar{y}_{i(N(i)-1)}\right)
\end{aligned}
$$

where $\sigma_{C}(\cdot, \cdot)$ is the sesquilinear form associated with the operator $L_{C}$.

With these definitions, we are able to write equation (17) in the usual weak form:

$$
<\hat{\rho} \ddot{W}(t), \Phi>+\hat{\sigma}_{C}(\dot{W}(t), \Phi)+\hat{\sigma}_{K}(W(t), \Phi)=0 \quad \text { for all } \Phi \in V \text {. }
$$

Since the boundary conditions for the continuous structure are assumed to be in such a form that the operators $L_{K}$ and $L_{C}$ are self-adjoint, it is easy to see that $\sigma_{K}$ and $\sigma_{C}$ are symmetric. Therefore, from the above definitions, it is easily verified that both $\hat{\sigma}_{K}$ and $\hat{\sigma}_{C}$ are symmetric, $V$-continuous and $V$-elliptic, from which the existence of a unique solution of equation (18) taken with appropriate initial conditions easily follows (again, see Chap. 4 of (Banks, Smith, and Wang, 1996))

Our primary concern is to use these sesquilinear forms to derive conditions under which there exist normal modes for system (17) (or (18)).

Theorem 3 Under assumptions (H1) and (H2), there exist normal modes for the discretecontinuous system (17) if and only if there exists a constant $k>0$ such that

$$
p_{K}=k p_{C}
$$

where

$$
p_{K}=\left(1, k_{1}, \cdots, k_{\mathcal{N}_{e}}, k_{11}, \cdots, k_{1 N(1)}, \cdots, k_{\mathcal{N}_{o} N\left(\mathcal{N}_{o}\right)}\right)
$$

and

$$
p_{C}=\left(\gamma, c_{1}, \cdots, c_{\mathcal{N}_{\epsilon}}, c_{11}, \cdots, c_{1 N(1)}, \cdots, c_{\mathcal{N}_{o} N\left(\mathcal{N}_{o}\right)}\right)
$$

Proof: The sufficiency is obvious. We need only to show the necessity. Let $\Phi_{n}(x)=$ $\left(w^{n}(x), z_{1}^{n}, \cdots, z_{\mathcal{N}_{o}}^{n}\right)^{T}$ be the generalized eigenfunctions of the operator $\hat{L}_{K}$ such that

$$
<\hat{\rho} \Phi_{n}, \Phi_{m}>=\delta_{n m}, \quad \text { for all } n, m .
$$

These eigenfunctions can be obtained using the same procedures as given in the Appendix. In order to have normal modes, it is necessary that

$$
\hat{\sigma}_{K}\left(\Phi_{n}, \Phi_{m}\right)=0
$$




$$
\hat{\sigma}_{C}\left(\Phi_{n}, \Phi_{m}\right)=0
$$

holds for any $n \neq m$. Assumption (H2) implies that

$$
\sigma_{C}\left(w^{n}, w^{m}\right)=\gamma \sigma_{K}\left(w^{n}, w^{m}\right)
$$

for a constant $\gamma$. Thus eq.(20) is equivalent to the following equations:

$$
\begin{gathered}
x_{0}^{n m}+\sum_{i=1}^{\mathcal{N}_{e}} k_{i} x_{i}^{n m}+\sum_{i=1}^{\mathcal{N}_{o}} k_{i 1} y_{i 1}^{n m}+\sum_{i=1}^{\mathcal{N}_{o}} k_{i 2} y_{i 2}^{n m}+\cdots+\sum_{i=1}^{\mathcal{N}_{o}} k_{i N(i)} y_{i N(i)}^{n m}=0 \\
\gamma x_{0}^{n m}+\sum_{i=1}^{\mathcal{N}_{e}} c_{i} x_{i}^{n m}+\sum_{i=1}^{\mathcal{N}_{0}} c_{i 1} y_{i 1}^{n m}+\sum_{i=1}^{\mathcal{N}_{o}} c_{i 2} y_{i 2}^{n m}+\cdots+\sum_{i=1}^{\mathcal{N}_{o}} c_{i N(i)} y_{i N(i)}^{n m}=0
\end{gathered}
$$

where

$$
\begin{aligned}
x_{0}^{n m} & =\sigma_{K}\left(w^{n}, w^{m}\right) \\
x_{i}^{n m} & =w^{n}\left(\xi_{i}\right) \bar{w}^{m}\left(\xi_{i}\right), \quad i=1, \cdots, \mathcal{N}_{e} \\
y_{i 1}^{n m} & =\left(z_{i 1}^{n}-w^{n}\left(\xi_{i}\right)\right)\left(\bar{z}_{i 1}^{m}-\bar{w}^{m}\left(\xi_{i}\right)\right), \quad i=1, \cdots, \mathcal{N}_{o} \\
& \vdots \\
y_{i N(i)}^{n m} & =\left(z_{i N(i)}^{n}-z_{i(N(i)-1)}^{n}\right)\left(\bar{z}_{i N(i)}^{m}-\bar{z}_{i(N(i)-1)}^{m}\right), \quad i=1, \cdots, \mathcal{N}_{o} .
\end{aligned}
$$

From eq.(21), we know that for each fixed $n$ and $m$

$$
v^{n m}=\left(x_{0}^{n m}, x_{1}^{n m}, \cdots, x_{\mathcal{N}_{e}}^{n m}, y_{11}^{n m}, \cdots, y_{\mathcal{N}_{o} 1}^{n m}, \cdots, y_{\mathcal{N}_{o} N\left(\mathcal{N}_{o}\right)}^{n m}\right)^{T}
$$

is orthogonal to $p_{K}$ when both of them are viewed as $1+\mathcal{N}_{e}+\sum_{i=1}^{\mathcal{N}_{o}} N(i)$ dimensional vectors.

We prove $p_{K}=k p_{C}$ by induction.

We have seen in Example 2 that when there is only one oscillator attached to the continuous member, $p_{K}=k p_{C}$ is necessary and sufficient in order to have normal modes. Example 3 shows also that $p_{K}=k p_{C}$ is necessary and sufficient for a system with one elastic support attached to the continuous member.

Consider then a system with $\mathcal{N}_{m}$ number of concentrated masses, $\mathcal{N}_{e}$ number of elastic supports, and $\sum_{i=1}^{\mathcal{N}_{0}} N(i)$ number of oscillators attached to the continuous member. Let $N=1+\mathcal{N}_{e}+\sum_{i=1}^{\mathcal{N}_{o}} N(i)$. Suppose that $p_{K}^{N}=k p_{C}^{N}$ is necessary for this system to have normal modes, where $p_{K}^{N}, p_{C}^{N}$ are the vectors $p_{K}, p_{C}$ for the $N$ dimensional system.

We are going to augment the above $N$-dimensional system into an $N+1$-dimensional augmented system. There are three ways to augment the system by one dimension.

1) Case 1: Attach one additional oscillator to an existing oscillator group, say, the $j$ 'th oscillator group, where $1 \leq j \leq \mathcal{N}_{\circ}$. 
2) Case 2: Attach one additional oscillator as a new oscillator group with only one oscillator, say, the $\left(\mathcal{N}_{0}+1\right)$ 'th oscillator group.

3) Case 3: Attach one additional elastic support, say, the $\left(\mathcal{N}_{\epsilon}+1\right)^{\prime}$ th elastic support to the continuous member.

We have to show that

$$
p_{K}^{N+1}=k p_{C}^{N+1}
$$

is necessary for this augmented system to have normal modes for each of the three cases above, where

$$
p_{K}^{N+1}=\left(p_{K}^{N}, p_{1}\right), \quad p_{C}^{N+1}=\left(p_{C}^{N}, p_{2}\right)
$$

and $p_{1}=k_{j(N(j)+1)}, p_{2}=c_{j(N(j)+1)}$ for case $1 ; p_{1}=k_{\left(\mathcal{N}_{\circ}+1\right) 1}, p_{2}=c_{\left(\mathcal{N}_{\circ}+1\right) 1}$ for case 2 ; and $p_{1}=k_{\left(\mathcal{N}_{e}+1\right)}, p_{2}=c_{\left(\mathcal{N}_{e}+1\right)}$ for case 3 .

We prove the necessity only for case 1 as an illustration; the other two cases can be similarly argued. Since we have that normal modes for the $N+1$ dimensional system implies normal modes for the $N$ dimensional system and hence $p_{K}^{N}=k p_{C}^{N}$, we need only to show $k_{j(N(j)+1)}=k c_{j(N(j)+1)}$ is also necessary. Suppose $k_{j(N(j)+1)} \neq k c_{j(N(j)+1)}$. Then it follows from equations (21)-(22) that

$$
\begin{aligned}
& \left(p_{K}^{N}, k_{j(N(j)+1)}\right) \cdot\left(v^{n m}, y_{j(N(j)+1)}^{n m}\right)=0, \\
& \left(p_{C}^{N}, c_{j(N(j)+1)}\right) \cdot\left(v^{n m}, y_{j(N(j)+1)}^{n m}\right)=0,
\end{aligned}
$$

where

$$
y_{j(N(j)+1)}^{n m}=\left(z_{j(N(j)+1)}^{n}-z_{j N(j)}^{n}\right)\left(\bar{z}_{j(N(j)+1)}^{m}-\bar{z}_{j N(j)}^{m}\right)
$$

Since $p_{K}^{N}=k p_{C}^{N}$, multiplying both sides of the second equation by $k$, and subtracting from the first we obtain

$$
\left(k_{j(N(j)+1)}-k c_{j(N(j)+1)}\right) y_{j(N(j)+1)}^{n m}=0 .
$$

If $k_{j(N(j)+1)}-k c_{j(N(j)+1)} \neq 0$, we have $y_{j(N(j)+1)}^{n m}=0$ for any $n \neq m$, which means

$$
z_{j(N(j)+1)}^{n}-z_{j N(j)}^{n}=0
$$

holds for all $n$, except possibly one $n_{1}$. Substituting this equation into the eigenvalue equation of $\hat{L}_{K}$, i.e.,

$$
\hat{L}_{K} \Phi_{n}=\lambda_{n} \Phi_{n}
$$

we see that

$$
z_{j(N(j)+1)}^{n}=z_{j N(j)}^{n}=\cdots=z_{j 1}^{n}=w^{n}\left(\xi_{j}\right)=0
$$


for all $n \neq n_{1}$, which is impossible since the eigenfunctions $\left\{w^{n}\right\}$ are determined a priori, once the physical system's configuration is determined. They cannot satisfy the constraint $w^{n}\left(\xi_{j}\right)=0$ for all $n \neq n_{1}$ and arbitrary $\xi_{j}$. This contradiction shows that $k_{j(N(j)+1)}=$ $k c_{j(N(j)+1)}$ is necessary for the augmented system to have normal modes. We have thus shown that (19) is a necessary and sufficient condition for the discrete-continuous system (17) to have normal modes.

When the relation in equation (19) holds, we say that the discrete-continuous systems are "matched". Since condition (19) is rarely satisfied, the two combined systems are most unlikely to be matched. Therefore, generically, the combined discrete-continuous systems do not have normal modes even if the continuous system alone does have normal modes.

It should also be remarked that the necessary and sufficient condition in Theorem 3 is a generalization of the well-known commutability condition. To see this, let us consider a situation where there is only one group of oscillators in a series connection. Let $n$ be the number of the oscillators, and let the spring coefficients and damping coefficients be respectively numbered as $k_{1}, k_{2}, \cdots, k_{n}$ and $c_{1}, c_{2}, \cdots, c_{n}$. Then from the definition of $\hat{L}_{K}$, we see that the stiffness operator in this case can be expressed as

$$
\begin{aligned}
& K^{n} z=\left(\begin{array}{c}
k_{1} z_{1}+k_{2}\left(z_{1}-z_{2}\right) \\
k_{2}\left(z_{2}-z_{1}\right)+k_{3}\left(z_{2}-z_{3}\right) \\
\vdots \\
k_{n}\left(z_{n}-z_{n-1}\right)
\end{array}\right) \\
& =\left(\begin{array}{cccc}
k_{1}+k_{2} & -k_{2} & & \\
-k_{2} & k_{2}+k_{3} & -k_{3} & \\
& -k_{3} & \ddots & \\
& & & k_{n}
\end{array}\right)\left(\begin{array}{c}
z_{1} \\
\vdots \\
z_{n}
\end{array}\right)
\end{aligned}
$$

and

$$
\begin{aligned}
& C^{n} z=\left(\begin{array}{c}
c_{1} z_{1}+c_{2}\left(z_{1}-z_{2}\right) \\
c_{2}\left(z_{2}-z_{1}\right)+c_{3}\left(z_{2}-z_{3}\right) \\
\vdots \\
c_{n}\left(z_{n}-z_{n-1}\right)
\end{array}\right) \\
& =\left(\begin{array}{cccc}
c_{1}+c_{2} & -c_{2} & & \\
-c_{2} & c_{2}+c_{3} & -c_{3} & \\
& -c_{3} & \ddots & \\
& & & c_{n}
\end{array}\right)\left(\begin{array}{c}
z_{1} \\
\vdots \\
z_{n}
\end{array}\right)
\end{aligned}
$$


It is well-known that a necessary and sufficient condition for this system (we assume without loss of generality that $\operatorname{diag}\left(m_{i}\right)=M=I$, i.e., these are the mass weighted stiffness and damping operators) to have normal modes is that $K^{n}$ and $C^{n}$ commute (Caughey, 1960), i.e.,

$$
K^{n} C^{n}=C^{n} K^{n}
$$

On the other hand, according to Theorem 3, we know that a necessary and sufficient condition is that there exists a scalar constant $k$ such that

$$
p_{K}^{n}=k p_{C}^{n},
$$

where

$$
\begin{aligned}
p_{K}^{n} & =\left(k_{1}, k_{2}, \cdots, k_{n}\right), \\
p_{C}^{n} & =\left(c_{1}, c_{2}, \cdots, c_{n}\right) .
\end{aligned}
$$

These two conditions are actually equivalent because of the following theorem.

Theorem 4. Let $K^{n}, C^{n}, p_{K}^{n}, p_{C}^{n}$ be defined as above. For any $n, K^{n} C^{n}=C^{n} K^{n}$ if and only if there exists a constant $k$ such that $p_{K}^{n}=k p_{C}^{n}$.

Proof. The sufficiency is obvious. We prove necessity by induction.

For $n=1$, this is obviously true. Suppose $K^{n} C^{n}=C^{n} K^{n}$ implies there exists a constant $k$ such that $p_{K}^{n}=k p_{C}^{n}$. We are going to show that $K^{n+1} C^{n+1}=C^{n+1} K^{n+1}$ implies $p_{K}^{n+1}=$ $k p_{C}^{n+1}$.

Since

$$
K^{n+1}=\left(\begin{array}{cc}
\tilde{K}_{11}^{n} & \tilde{K}_{12} \\
\tilde{K}_{12}^{T} & k_{n+1}
\end{array}\right)
$$

where

$$
\tilde{K}_{11}^{n}=K^{n}+\left(\begin{array}{cc}
0 & 0 \\
0 & k_{n+1}
\end{array}\right), \quad \hat{K}_{12}=\left(\begin{array}{c}
0 \\
-k_{n+1}
\end{array}\right),
$$

where 0 represents various zero matrices or vectors with compatible dimensions. Similarly,

$$
C^{n+1}=\left(\begin{array}{cc}
\tilde{C}_{11}^{n} & \tilde{C}_{12} \\
\tilde{C}_{12}^{T} & c_{n+1}
\end{array}\right)
$$

where

$$
\tilde{C}_{11}^{n}=C^{n}+\left(\begin{array}{cc}
0 & 0 \\
0 & c_{n+1}
\end{array}\right), \quad \tilde{C}_{12}=\left(\begin{array}{c}
0 \\
-c_{n+1}
\end{array}\right) .
$$


Therefore,

$$
K^{n+1} C^{n+1}=\left(\begin{array}{cc}
\tilde{K}^{n} \tilde{C}^{n}+\left(\begin{array}{cc}
0 & 0 \\
0 & k_{n+1} c_{n+1}
\end{array}\right) & \tilde{K}^{n} \tilde{C}_{12}+\tilde{K}_{12} c_{n+1} \\
\tilde{K}_{12}^{T} \tilde{C}^{n}+\tilde{C}_{12}^{T} k_{n+1} & 2 k_{n+1} c_{n+1}
\end{array}\right)
$$

and

$$
C^{n+1} K^{n+1}=\left(\begin{array}{cc}
\tilde{C}^{n} \tilde{K}^{n}+\left(\begin{array}{cc}
0 & 0 \\
0 & k_{n+1} c_{n+1}
\end{array}\right) & \tilde{C}^{n} \tilde{K}_{12}+\tilde{C}_{12} k_{n+1} \\
\tilde{C}_{12}^{T} \tilde{K}^{n}+\tilde{K}_{12}^{T} c_{n+1} & 2 k_{n+1} c_{n+1}
\end{array}\right)
$$

Comparing elements of these two matrices, we have

$$
\tilde{K}^{n} \tilde{C}_{12}=\tilde{C}^{n} \tilde{K}_{12},
$$

from which we obtain

$$
k_{n} c_{n+1}=c_{n} k_{n+1} .
$$

Since it is assumed that $k_{n}=k c_{n}$, we see from the above equation that $k_{n+1}=k c_{n+1}$. Therefore, $p_{K}^{n+1}=k p_{C}^{n+1}$. The proof is complete.

\section{Concluding Remarks}

In this paper, a general model has been derived for a combined elastic continuous structure and a finite number of discrete systems, with the objective to study conditions under which the combined system has normal modes. It is shown that unless a matching condition is satisfied, the system does not have normal modes even for Kelvin-Voigt damping. The matching condition is, however, very difficult to satisfy. Therefore, the combined systems rarely have normal modes even if the simple continuous system alone has normal modes. Note that this result discusses the nature of the zeros or eigenvectors of a transfer function of the system, not the poles. Hence the results apply to underdamped, overdamped and critically damped modes (i.e., complex, real, distinct or repeated poles).

We further note that our detailed analysis depends heavily on the form of damping which is assumed proportional (Kelvin-Voigt damping). However our techniques readily generalize to imply similar conclusions about the lack of normal modes for general classes of proportionally damped systems, including those with Rayleigh damping.

In our treatment we have used the standard definition of "normal modes" that requires zero off-diagonal terms in modal approximations. Issues related to approximation of nonnormal mode systems (i.e., those with nonzero off-diagonal elements) by normal mode (zero

off-diagional) approximations have been discussed for example in (Bellos, 1989; Bellos and Inman, 1989; Huang and Ma, 1994; Ma and Caughey, 1995). 


\section{Appendix}

We indicate how to solve the eigenvalue problem of the operator $A$ defined in Section 2. Equivalently, we want to solve for $\lambda_{n}, w_{n}(x)$ and $z_{n}$ from system (10) which is recapped as follows for convenience:

$$
\begin{gathered}
E I w_{n}^{\prime \prime \prime \prime}(x)+k_{H}\left(w_{n}(\alpha)-z_{n}\right) \delta(x-\alpha)=\rho \lambda_{n} w_{n}(x), \quad 0<x<\ell \\
k_{H}\left(z_{n}-w_{n}(\alpha)\right)=m_{H} \lambda_{n} z_{n} \\
w_{n}(0)=w_{n}^{\prime}(0)=w_{n}^{\prime \prime}(\ell)=w_{n}^{\prime \prime \prime}(\ell)=0 .
\end{gathered}
$$

Since a delta function is contained in this equation, we may consider the problem on two separate regions $\left(0, \alpha^{-}\right]$and $\left[\alpha^{+}, \ell\right)$. For $0<x \leq \alpha^{-}$, system $(23)$ reduces to

$$
\begin{gathered}
E I w_{n}^{\prime \prime \prime \prime}(x)=\rho \lambda_{n} w_{n}(x), \quad 0<x<\alpha^{-} \\
k_{H}\left(z_{n}-w_{n}\left(\alpha^{-}\right)\right)=m_{H} \lambda_{n} z_{n}
\end{gathered}
$$

and for $\alpha^{+} \leq x<\ell$, system (23) reduces to

$$
\begin{gathered}
E I w_{n}^{\prime \prime \prime \prime}(x)=\rho \lambda_{n} w_{n}(x), \quad \alpha^{+}<x<\ell \\
k_{H}\left(z_{n}-w_{n}\left(\alpha^{+}\right)\right)=m_{H} \lambda_{n} z_{n} .
\end{gathered}
$$

At the point $x=\alpha$, the derivatives of $w_{n}(x)$ up to the 2 nd-order must be continuous, so that

$$
\begin{aligned}
& w_{n}\left(\alpha^{-}\right)=w_{n}\left(\alpha^{+}\right), \\
& w_{n}^{\prime}\left(\alpha^{-}\right)=w_{n}^{\prime}\left(\alpha^{+}\right), \\
& w_{n}^{\prime \prime}\left(\alpha^{-}\right)=w_{n}^{\prime \prime}\left(\alpha^{+}\right) .
\end{aligned}
$$

Integration of the first equation in system (23) yields

$$
E I w_{n}^{\prime \prime \prime}\left(\alpha^{+}\right)-E I w_{n}^{\prime \prime \prime}\left(\alpha^{-}\right)=k_{H}\left(z_{n}-w_{n}(\alpha)\right)
$$

which is the jump condition for the shear force. Substituting the second equation of system (23) to eliminate $z_{n}$ in equation (27), we obtain

$$
E I w_{n}^{\prime \prime \prime}\left(\alpha^{+}\right)-E I w_{n}^{\prime \prime \prime}\left(\alpha^{-}\right)=\frac{k_{H} m_{H} \lambda_{n}}{k_{H}-m_{H} \lambda_{n}} w_{n}(\alpha) .
$$

In addition to these conditions, $w_{n}(x)$ should of course satisfy the boundary conditions at both ends $x=0$ and $x=\ell$, i.e.,

$$
w_{n}(0)=w_{n}^{\prime}(0)=w_{n}^{\prime \prime}(\ell)=w_{n}^{\prime \prime \prime}(\ell)=0 .
$$


Let $\lambda_{n}=E I \beta_{n}^{4}$. Then the solution of equation (24) is given by

$$
w_{n}(x)=C_{1} e^{\beta_{n} x}+C_{2} e^{-\beta_{n} x}+C_{3} e^{j \beta_{n} x}+C_{4} e^{-j \beta_{n} x}, \quad 0<x<\alpha
$$

and the solution of equation (25) is given by

$$
w_{n}(x)=C_{5} e^{\beta_{n} x}+C_{6} e^{-\beta_{n} x}+C_{7} e^{j \beta_{n} x}+C_{8} e^{-j \beta_{n} x}, \quad \alpha<x<\ell
$$

where $C_{i}, i=1, \cdots, 8$ are unknown constants to be determined. Since there are exactly 8 boundary conditions as shown in eqs.(26),(28), (29), there exists a unique solution for $C_{i}$ when $\lambda_{n}$ satisfies some specific algebraic equation from which we can evaluate the eigenvalues. Once the $C_{i}$ are determined, the eigenfunctions $w_{n}(x), 0<x<\ell$ are readily obtained from equations $(30)$ and $(31)$.

\section{Acknowledgment:}

This research was supported by the U.S. Air Force Office of Scientific Research in part (H.T.B.) under grant AFOSR F49620-95-1-0236 and in part (H.T.B, D.J.I.) under grant AFOSR F49620-93-1-0280. The second author would like to gratefully acknowledge Prof. K. Ito for numerous discussions on a number of topics while he was a visiting professor at N.C. State University.

\section{References}

Banks, H.T., Gates, S.S., Rosen, I.G., and Wang, Y., 1988, "The identification of a distributed parameter model for a flexible structure," SIAM J. Control and Optimization, vol. 26 , pp. $743-762$.

Banks, H.T., Smith, R.C., and Wang, Y., 1996, Smart Material Structures: Modeling, Estimation and Control, Masson/Wiley, Paris/Chichester.

Bellos, J., 1989, Theoretical and Experimental Analysis of Non-Proportional Damping, PhD Dissertation, State University of New York at Buffalo, Department of Mechanical and Aerospace Engineering.

Bellos, J. and Inman, D.J., 1989, "A survey on non proportional damping," Shock and Vibration Digest, vol. 27, no. 10, pp. 7-12.

Bergman, L.A. and McFarland, D.M., 1988, "On the vibration of a point supported linear distributed system," ASME Journal of Vibration, Acoustics, Stress, and Reliability in Design, vol. 110, pp. 485-492. 
Bergman, L.A. and McFarland, D.M., 1988 "Vibration of a class of complex discretedistributed systems," Proc. of the 29th AIAA Structures, Structural Dynamics and Materials Conference, pp. 890-900.

Bergman, L.A. and Nicholson, J.W., 1985, "Forced vibration of damped combined linear system," ASME Journal of Vibration, Acoustics, Stress, and Reliability in Design, vol. 107, pp. $275-281$.

Bergman, L.A. and Nicholson, J.W., 1985, "On the free and forced torsional vibration of multi-disk shaft systems," Proc. of the 26th AIAA Structures, Structural Dynamics and Materials Conference, pp. 515-521.

Bishop, R.E.D. and Johnson, D.C., 1960, The Mechanics of Vibration, Cambridge University Press.

Caughey,T.K., 1960, "Classical normal modes in damped linear dynamic systems," ASME Journal of Applied Mechanics, vol. 27, no. 2, pp. 269-271.

Caughey, T.K. and O‘Kelley, M.E.J., 1965, "Classical normal modes in damped linear systems," ASME Journal of Applied Mechanics, vol. 32, no. 3, pp. 583-588.

Chen, Y., 1963, "On beams with many elastic supports," Journal of the Franklin Institute, vol. 276 , no. 4, pp. $273-281$.

Chen, Y., 1963, "On the vibration of beams or rods carrying a concentrated mass," ASME Journal of Applied Mechanics, vol. 30, no. 2, pp. 310-311.

Davies, H.G. and Rogers, R.J., 1979, "The vibration of structures elastically restrained at discrete points," Journal of Sound and Vibration, vol. 63, no. 3, pp. 437-447.

Dowell, E.H., 1979, "On some general properties of combined dynamical systems," ASME Journal of Applied Mechanics, vol. 46, pp. 206-209.

Ewins, D.J., 1984, Modal Testing Theory and Practice, Research Studies Press.

Gupta, G.S., 1970, "Natural flexural waves and the normal modes of periodically supported beams and plates," Journal of Sound and Vibration, vol. 13, no. 1, pp. 89-101.

Huang, J.H. and Ma, F., 1994 "On the approximate solution of nonclassically damped linear systems," ASME Journal of Applied Mechanics, vol.60, pp. 695-701.

Inman, D..J., 1989, Vibration: With Control Measurement and Stability, Prentice Hall, New Jersey.

Inman, D.J. and Olsen, C.L., 1988, "Dynamics of symmetrizable nonconservative systems," ASME Journal of Applied Mechanics, vol. 55, pp. 206-212.

Jacquot, R.G., 1970, "Vibrations of elastic surface systems carrying dynamic elements," Journal of the Acoustical Society of America, vol. 47, no. 5, Part 2, pp. 1354-1358.

Kasprzyk, S., 1984, "An analysis of vibrations of a discrete-continuous system of the type $(\infty, 2), "$ Nonlinear Vibration Problems, vol. 22, pp. 199-214. 
Lin, Y.K., 1962, "Free vibrations of a continuous beam on elastic supports," Int. Journal of the Mechanical Sciences, vol. 4, pp. 400-423.

McFarland, D.M., 1990, Analysis of Passive and Active Discrete-Distributed Linear Dynamical Systems Using Green's Function Methods, PhD Dissertation, Dept. of Aeronautical and Astronautical Engineering, University of Illinois.

Ma, F. and Caughey, T.K., 1995, "Analysis of linear nonconservative systems," ASME Journal of Applied Mechanics, vol. 62, pp. 685-691.

Mead, D.J., 1971, "Vibration response and wave propagation in periodic structures," ASME Journal of Engineering for Industry, pp.783-792.

Meirovitch, L., 1967, Analytical Methods in Vibrations, Macmillan.

Miles, J.W., 1956, "Vibrations of beams on many supports," ASCE Journal of the Engineering Mechanics Division, pp. 1-9.

Nicholson, J.W. and Bergman, L.A., 1986, "Free vibration of combined dynamical systems," ASCE Journal of Engineering Mechanics, vol. 112, no. 1, pp. 1-13.

Nicholson, J.W. and Bergman, L.A., 1986 "Vibration of damped plate-oscillator systems," ASCE Journal of Engineering Mechanics, vol. 112, no. 1, pp. 14-30.

Nicholson, J.W. and Bergman, L.A., 1985, "Vibration of thick plates carrying concentrated masses," Journal of Sound and Vibration, vol. 103, no. 3, pp. 357-369.

Pan, H.H., 1965, "Transverse vibration of an Euler beam carrying a system of heavy bodies," ASME Journal of Applied Mechanics, vol. 32, no. 2, pp.434-437.

Wloka, J., 1992, Partial Differential Equations, Cambridge University Press, Cambridge. 